\title{
Nutritional status alters the lipidome of a bethylid wasp incapable of de novo lipogenesis
}

\author{
C.J.P. Snart ${ }^{1,2}$, A. Kapranas ${ }^{1}$, D.A. Barrett ${ }^{2}$ and I.C.W. Hardy ${ }^{1}$ \\ ${ }^{1}$ Division of Agricultural and Environmental Sciences, School of Biosciences, University of Nottingham, LE12 5RD, \\ $U K$ and ${ }^{2}$ Centrefor Analytical Bioscience, School of Pharmacy, University of Nottingham, NG7 2RD, UK
}

Physiological status is greatly influenced by the quality of available nutrient uptake. Nutrient acquisition strategies vary across insect parasitoids, including host feeding and the ingestion of environmental sugars ${ }^{(1)}$. Hymenopteran parasitoids do not possess de novo lipogenesis ${ }^{(2)}$ and are limited to lipids obtained during larval development. However, parasitoid lifespan and fecundity is enhanced by carbohydrate intake ${ }^{(3)}$. Using a metabolomics approach and liquid chromatography-mass spectrometry (LC-MS) we aim to assess the effects of dietary treatments on the lipidome of the parasitoid wasp Goniozus legneri.

Adult female $G$. legneri were isolated and either starved or provided with a honey diet for 7 days $(n=20$ per treatment), then frozen in liquid nitrogen. Individual wasps were extracted using a modified methanol-chloroform-water solvent methodology and concentrated prior to LC-MS analysis. Fragmentation patterns of differential lipids were generated using LC-MS-MS to aid identification. Differentiation between classes was determined by combined PCA/OPLS-DA and validated with a one-way logistic ANOVA.

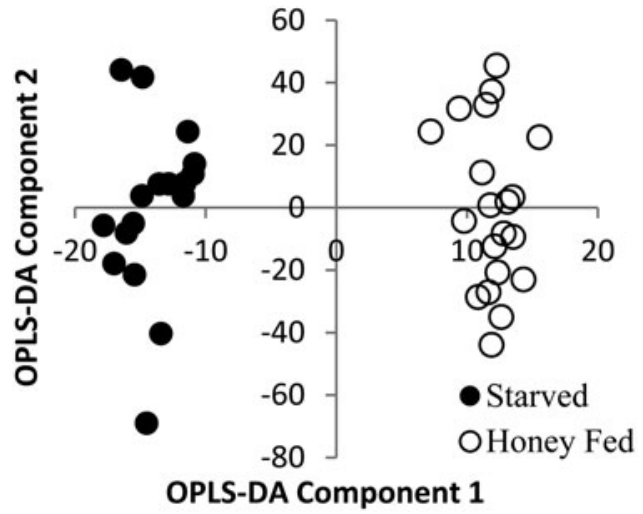

Fig. 1. OPLS-DA analysis of MS samples of honey-fed and starved G. legneri.

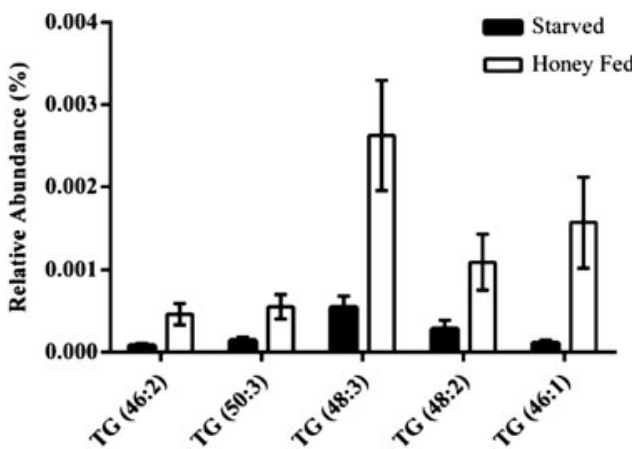

Fig. 2. Differential triglyceride levels between honey-fed and starved G. legneri.

A total of 38 differential ions were identified in positive ESI mode (logistic ANOVA with Bonferoni correction: $\mathrm{P}<0 \cdot 05$, df $=2,37$ ), consisting of 37 upregulated amongst honey-fed and 1 in starved G. legneri. Differential lipids strongly associated with honey-fed wasps were large storage glycerolipids, including 25 unique triglycerides. Remaining honey-treatment associated differential lipids are comprised of digycerides and phospholipids. The single upregulated lipid in starved wasp samples was identified as a phosphocholine. In conclusion, adult female $G$. legneri can utilise simple carbohydrates as an energy source, and as a result, may be capable of preserving storage lipids for maintenance and egg allocation.

This work was supported by the BBSRC and a Marie Curie fellowship awarded by the European Commission.

1. Olson DM, Fadamiro H, Lundgren JG and Heimpel GE (2000) Physiol Entomol 25, 17-26.

2. Visser B and Ellers J (2008) J Insect Physiol 54, 1315-1322.

3. Ellers (1996) Neth $J$ Zool 46, 227-235. 